\title{
BIBLIOTECA ESCOLAR E LETRAMENTO NO CONTEXTO DO SEMIÁRIDO: UMA ANÁLISE A PARTIR DAS POLÍTICAS PÚBLICAS EM ESCOLAS DE JUAZEIRO-BA: CAMINHOS METODOLÓGICOS
}

\author{
Tatiane Lemos Alves ${ }^{1}$ \\ Cosme Batista dos Santos ${ }^{2}$
}

\begin{abstract}
Resumo
Este trabalho tem por objetivo apresentar as questões metodológicas utilizadas para realizar uma pesquisa que visou investigar o papel da biblioteca na formação dos leitores em escolas públicas da cidade de Juazeiro-BA. Primeiramente, discutiremos a escolha do estudo de caso como estratégia de pesquisa. Em seguida, refletiremos sobre a abordagem quali-quantitativa no processo investigatório. $\mathrm{E}$, por fim, indicamos os instrumentos utilizados para construção de dados, tais como observação das práticas letradas, entrevista com integrantes da comunidade escolar, análise de documentos norteadores das bibliotecas, além da proposta de mapeamento das produções cientificas, estabelecendo o estado da arte sobre biblioteca escolar, nos artigos, dissertações e teses no período de 2010 a 2015 em bases de dados brasileiras. A análise quali-quantitativa das políticas públicas sobre leitura, livros e biblioteca possibilitou que concluíssemos que boas práticas já se iniciaram no ambiente das bibliotecas selecionadas com apoio das políticas públicas, e que apesar das dificuldades encontradas, a biblioteca escolar tem papel importante para a formação dos leitores nas escolas e na comunidade externa; contudo, ainda, tem algumas lacunas que precisam ser sanadas, tais como a necessidade de capacitação, avaliação periódica das políticas além de maior entrosamento dos atores envolvidos, para potencializar e multiplicar as iniciativas positivas visando melhor atender à comunidade escolar e formar leitores críticos e autônomos.
\end{abstract}

Palavras-chave: Metodologia. Pesquisa. Biblioteca escolar. Políticas públicas.

\footnotetext{
${ }^{1}$ Mestre em Educação, Cultura e Territórios Semiáridos - PPGESA/ UNEB. Especialista em Metodologia e Didática do Ensino Superior. Bacharel em Biblioteconomia e Documentação pela UFBA. Bibliotecária no Instituto Federal do Sertão Pernambucano.

${ }^{2}$ Pós-doutor em Ciência da Informação pela Faculdade de Letras da Universidade do Porto (FLUP) e Estágio Sênior/CAPES em Lexiculturologia pela Universidade Católica Portuguesa (UCP), de Braga. Doutor e mestre em Linguística Aplicada pelo Instituto de Estudos da Linguagem da Universidade Estadual de Campinas - UNICAMP. Possui graduação em Letras pela Universidade de Pernambuco UPE (1992), especialização em Programação de Ensino de Língua Portuguesa e Literatura pela Universidade de Pernambuco - UPE (1993) e especialização em Língua Falada e Ensino pela Pontifícia Universidade Católica de Minas Gerais - PUC Minas (1995). Atualmente é Professor Pleno da Universidade do Estado da Bahia, atuando no Programa de Pós-graduação em Critica Cultural POSCRÍTICA e no Programa de Pós-graduação em Educação, cultura e territórios semiáridos PPGESA.
} 


\title{
SCHOOL LIBRARY AND LETTERING IN THE SEMIARID CONTEXT: AN ANALYSIS FROM PUBLIC POLICIES IN JUAZEIRO-BA SCHOOLS: METHODOLOGICAL PATHWAYS
}

\begin{abstract}
This paper aims to present the methodological questions involved in a research project that investigated the role of the library in the training of readers in public schools in the city of Juazeiro. First, we will discuss the choice of the case study as a research strategy. Next, we will reflect on the qualitative-quantitative approach in the investigative process. Finally, we indicate the tools used to construct data, such as observation of literate practices, interview with members of the school community, analysis of documents that guide the libraries, as well as mapping the scientific productions, establishing the state of the art on school library research through articles, dissertations and theses in the period from 2010 to 2015 in Brazilian databases. The qualitative and quantitative analysis of public policies on reading, books and the library allowed us to conclude that good practices have already begun in the environment of selected libraries with the support of public policies, and that despite the difficulties encountered, the school library plays an important role in the formation of readers in schools and in the external community; however, there are some shortcomings that need to be addressed, such as the need for training, periodic evaluation of policies and more involvement of the actors involved, to increase and multiply positive initiatives aimed at better serving the school community and training critical and autonomous readers .
\end{abstract}

Keywords: Methodology. Search. School library. Public policy.

\section{BIBLIOTECA ESCOLAR Y LITERACIDAD EN EL CONTEXTO SEMIÁRIDO: UN ANÁLISIS DE LAS POLÍTICAS PÚBLICAS EN LAS ESCUELAS JUAZEIRO-BA: CAMINOS METODOLÓGICOS}

\section{Resumen}

Este trabajo tiene por objetivo presentar las cuestiones metodológicas utilizadas para realizar una investigación que pretendía investigar el papel de la biblioteca en la formación de lectores en escuelas públicas de la ciudad de Juazeiro-BA. Primero, discutiremos la elección del estudio de caso como estrategia de investigación. A continuación, reflexionaremos sobre el enfoque cualitativo cuantitativo en el proceso de investigación. Y por último, indicamos los instrumentos utilizados para la construcción de datos, tales como observación de las prácticas letradas, entrevista con integrantes de la comunidad escolar, análisis de documentos orientadores de las bibliotecas además de mapear las producciones científicas, estableciendo el estado del arte sobre biblioteca escolar, artículos, disertaciones y tesis en el período 2010 a 2015 en bases de datos brasileñas. El análisis cuali cuantitativo de las políticas públicas sobre lectura, libros y biblioteca posibilitó que concluyamos que buenas prácticas ya se iniciaron en el ambiente de las bibliotecas seleccionadas con apoyo de las políticas públicas, y que a pesar de las dificultades encontradas, la biblioteca escolar juega un papel importante para la formación de los lectores en las escuelas y en la comunidad externa; sin embargo, tiene algunas deficiencias que necesitan ser subsanadas, tales como la 
necesidad de capacitación, evaluación periódica de las políticas además de mayor integración de los actores involucrados, para potenciar y multiplicar las iniciativas positivas con el objetivo de atender mejor a la comunidad escolar y formar lectores críticos y autónomos .

Palabras clave: Metodología. La investigación. Biblioteca escolar. Políticas públicas.

\section{Introdução}

Este artigo é parte de um trabalho dissertativo que teve como foco a biblioteca escolar considerando o importante papel desempenhado por esta, principalmente ao apoiar o currículo, promover a aprendizagem e oportunizar a democratização cultural, através do acesso ao mundo da leitura. Essas reflexões são bastante pertinentes em se tratando de Semiárido Brasileiro, pela possibilidade de essa biblioteca poder vir a ser instrumento de uma educação com mais pluralidade, que considera os valores, concepções e potencialidades desse contexto. Sendo assim, a pesquisa buscou investigar o papel da biblioteca na formação dos leitores em escolas públicas da cidade de Juazeiro-BA. Particularmente, este artigo traz contribuições para as pesquisas sobre o papel da biblioteca no letramento escolar, em especial, em relação às condições metodológicas de investigação para potencializar práticas bem sucedidas de gestão de bibliotecas, tendo em vista o destaque dessas práticas e das políticas de leitura na educação básica em geral.

Para tanto, utilizou-se como base metodológica a abordagem quali-quantitativa visando mapear as produções científicas, estabelecendo o estado da arte sobre biblioteca escolar, nos artigos, dissertações e teses no período de 2010 a 2015; traçou-se o perfil dos usuários das bibliotecas escolares; identificou-se as práticas desenvolvidas no ambiente das bibliotecas; além de verificar-se a existência da articulação entre as práticas de leitura desenvolvidas pelos professores com as da biblioteca.

O foco deste trabalho, particularmente, é apresentar detalhadamente a opção metodológica utilizada para alcançar os objetivos da dissertação já mencionados, como veremos a seguir. Assim, este artigo está organizado da seguinte forma: a próxima seção apresenta as escolhas metodológicas, desde o estudo de caso, passando pela abordagem da pesquisa e indicando os instrumentos empregados na construção de dados. E, por fim, reflete as opções escolhidas nas considerações gerais. 


\section{Caminhos metodológicos}

Um dos elementos basilares para a orientação de qualquer trabalho científico é a escolha metodológica, constituindo-se a escolha de procedimentos e/ou estratégias para o desenvolvimento da pesquisa. Nesta pesquisa, optou-se pelo uso do estudo de caso como estratégia de pesquisa.

A metodologia de investigação apoiou-se no estudo de caso (YIN, 2010), seguindo uma abordagem quali-quantitativa, visto que nos preocupamos em compreender as diversas faces que envolvem um fenômeno social. Os instrumentos que foram utilizados para a construção dos dados foram a observação participante, a entrevista e a análise documental. O lócus de pesquisa foi delineado a partir de um levantamento nas Instituições educacionais da Rede Estadual de Ensino da cidade de Juazeiro-BA, identificando quais possuíam biblioteca; em seguida, foram selecionadas as escolas que estavam em pleno funcionamento, com espaço específico para as atividades da biblioteca, com funcionário encarregado e que estivesse dentro do perímetro territorial preestabelecido. Posteriormente, concentramos a investigação nas duas bibliotecas que atendiam a todos os critérios preestabelecidos, as do Colégio Estadual Artur Oliveira da Silva e da Escola Mandacaru.

\section{Estudo de caso}

O estudo de caso é uma investigação empírica, que explora um fenômeno contemporâneo em profundidade e em seu contexto de vida real, especialmente quando os limites entre o fenômeno e o contexto não são claramente evidentes. (YIN, 2010) Por extensão, pode apresentar variações, nas quais inclui um único caso ou múltiplos casos. Os estudos de caso são normalmente aqueles que focalizam apenas uma unidade de análise, seja um indivíduo, um grupo, uma instituição, um evento ou programa. Todavia, existem também estudos de casos múltiplos, em que vários estudos são conduzidos simultaneamente, ou seja, com vários indivíduos ou várias instituições. $\mathrm{Na}$ presente investigação, adotou-se o estudo de casos múltiplos, pois foram escolhidas duas bibliotecas de instituições de ensino públicas da cidade de Juazeiro-BA.

Quanto ao delineamento da pesquisa, Yin (2010, p.41) elucida que "os estudos de caso podem incluir detalhes e até mesmo ser limitados à evidência quantitativa.” Ele, ainda, acrescenta que “o estudo de caso não é apenas uma forma de 'pesquisa 
qualitativa', mesmo que possa ser reconhecida entre a variedade de opções da pesquisa qualitativa”. Algumas opções metodológicas vão além de um tipo de pesquisa específico, usando a mistura de quantitativa e qualitativa em que as evidências quantitativas pode ser tratadas qualitativamente, tal como é a opção metodológica da pesquisa que estamos apresentando neste artigo.

\section{Abordagem da pesquisa}

A escolha da abordagem de pesquisa se deu no sentido de compreender de forma mais completa os dados coletados e na intenção de refletir a dinâmica da teoria. Logo se optou por utilizar tanto a abordagem qualitativa quanto quantitativa de forma complementar, como já informamos no tópico anterior.

Sobre o uso das abordagens qualitativa e quantitativa na mesma pesquisa, Minayo e Sanches (1993, p. 247) indicam que elas podem e devem ser utilizadas, em tais circunstâncias, como complementares, visto que, segundo os autores, não há contradição entre as abordagens, posto que a primeira "trabalha com valores, crenças, representações, hábitos, atitudes e opiniões", e a segunda "tem como campo de práticas e objetivos trazer à luz dados, indicadores e tendências observáveis".

Corroborando com esse pensamento, Goldenberg (2004, p.62) explica que, com a junção das abordagens, “o pesquisador tem condições para produzir um conhecimento completo da realidade, pois diferentes abordagens de pesquisa podem projetar luz sobre diferentes questões". Dessa forma, o conjunto de diferentes pontos de vista e diferentes maneiras de coletar e analisar os dados (qualitativa e quantitativamente) permite uma ideia mais ampla e inteligível da complexidade de um problema, pois essa integração permite que o pesquisador faça um cruzamento dos dados de modo a ter maior confiança. (GOLDENBERG, 2004).

A pesquisa em questão, portanto, revelou a preocupação em diagnosticar um fenômeno, ou seja, descrevê-lo e interpretá-lo, além de explicá-lo a partir de seus determinantes, o que característica a utilização da abordagem quali-quantitativa. Ainda no universo desta pesquisa, utilizou-se a complementaridade das duas abordagens, posto que uma possibilitou o aprofundamento da compreensão das condições de acesso à leitura na escola, assim como as práticas desenvolvidas nas bibliotecas e a outra permitiu a mensuração de indicadores relacionados ao perfil dos usuários das 
bibliotecas. Logo, a utilização conjunta da pesquisa qualitativa e quantitativa permitiu recolher mais informações do que se poderia conseguir com as abordagens isoladamente.

Nesse caminhar, no que diz respeito à organização do trabalho de campo, foram selecionados três instrumentos para o desenvolvimento da pesquisa, a saber: observação participante, entrevista e análise documental.

\section{Instrumentos de construção de dados}

A utilização da entrevista e da observação como instrumentos de coleta de dados permite comparações entre os discursos e a ação dos participantes da pesquisa. A análise documental foi utilizada para validação das informações obtidas durante a entrevista com os informantes das bibliotecas pesquisadas. Além disso, a pesquisa foi subsidiada com o mapeamento e discussão da produção acadêmica em diferentes campos do conhecimento através da constituição do Estado da Arte.

\section{Observação participante}

Foto 1 - Observação participante

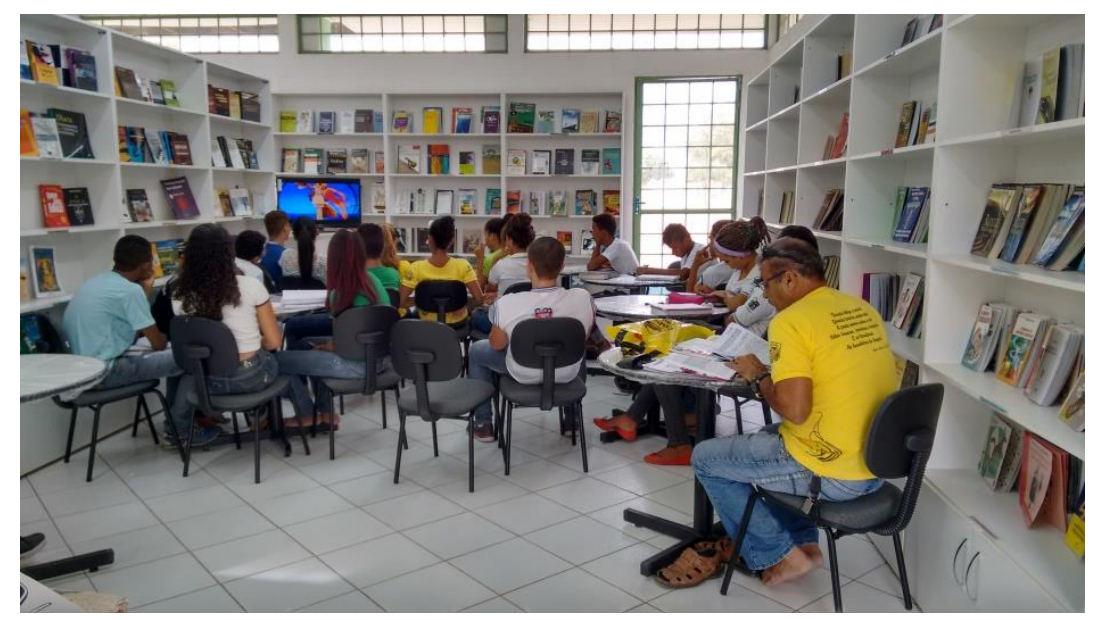

Foto 1: Tatiane Alves

A observação, assim como a entrevista, merece destaque nas pesquisas educacionais, pois possibilita um contato pessoal e estreito do pesquisador com o fenômeno pesquisado (LUDKE e ANDRÉ,1986). Neste estudo, a observação se deu em dois momentos, o primeiro período de setembro a novembro de 2015, em que foram 
observadas as primeiras informações acerca das escolas e suas bibliotecas, e o segundo, de maio a setembro de 2016, nas duas escolas selecionadas para aprofundamento do estudo.

Segundo Gil (2008, p. 101), “a observação nada mais é que o uso dos sentidos com vistas a adquirir os conhecimentos necessários para o cotidiano". Essa pode ser classificada, segundo o grau de participação do observador, assumindo, assim, duas formas, a participante ou não participante. Pela natureza desta investigação, a pesquisadora se posicionou-se como uma observadora participante, para obter maior conhecimento sobre o cotidiano dos frequentadores das bibliotecas, tendo em vista a participação real da pesquisadora nessas comunidades. A escolha por esse instrumento se deu por ele possibilitar o contato direto com as pessoas, os acontecimentos e as situações dentro do ambiente natural estudado. Entende-se a importância da observação participante para o desenvolvimento da pesquisa, uma vez que a ação permite entender as práticas no ambiente das bibliotecas e quebra o distanciamento entre os sujeitos da pesquisa e o pesquisador.

De acordo, com André (2010), a observação é chamada de participante porque parte do princípio de que o pesquisador tem sempre um grau de interação com a situação estudada, afetando-a e sendo por ela afetado. Já para Oliveira (2013, p. 82), na observação participante, existe uma análise descritiva do fato ou fenômeno observado; uma delimitação dos fatos a serem observados, segundo o objeto de estudo e, por fim, uma seleção dos dados a serem analisados, segundo a delimitação feita na etapa anterior.

Diante do exposto, a observação participante realizada durante oito meses permitiu que a pesquisadora chegasse mais perto da perspectiva dos participantes da pesquisa, na medida em que a investigação acompanhou in loco as experiências diárias das pessoas envolvidas, estando e observando onde a ação acontecia visando a atender aos objetivos da pesquisa. Para tanto, foi utilizado um roteiro que permitiu examinar a situação, buscando descrevê-la usando a nossa interpretação acerca daquele fenômeno.

\section{Entrevista}

Para complementar os dados coletados na observação, foi necessária a realização de entrevistas que, nesta pesquisa, tiveram como foco identificar as práticas desenvolvidas no ambiente das bibliotecas, a partir da perspectiva dos usuários e dos 
responsáveis pelas bibliotecas além de verificar se existia articulação entre as práticas de leitura desenvolvidas pelos professores com as atividades desenvolvidas na biblioteca. De acordo com Gil (2008, p. 109), utilizar esse instrumento é uma forma de interação social, na qual o investigador se apresenta frente ao investigado e lhe formula perguntas, com o objetivo de obtenção dos dados que interessam à investigação. Assim, elas "podem ser utilizadas para investigar um tema em profundidade, como ocorre nas pesquisas designadas como qualitativas" (GIL, 2008, p.114).

Nesse sentido, a entrevista foi adotada pela possibilidade de interação entre o entrevistado e a pesquisadora além da oportunidade de captação imediata da informação desejada. Para esta investigação, utilizou-se a entrevista semiestruturada, que permite a elaboração de um roteiro de perguntas para nortear a condução da entrevista junto ao entrevistado. Além disso, destaca-se a liberdade de adaptar as perguntas ou alterar a ordem dos tópicos sem prejuízo ao produto final. Foram entrevistados vinte e dois sujeitos, sendo dezesseis estudantes e seis professores, a fim de aprofundar a análise daqueles que utilizam a biblioteca. As conversas foram gravadas em áudio com anuência dos sujeitos entrevistados e posteriormente, transcritas. ${ }^{3}$

Foto 2 - Práticas de leitura na biblioteca

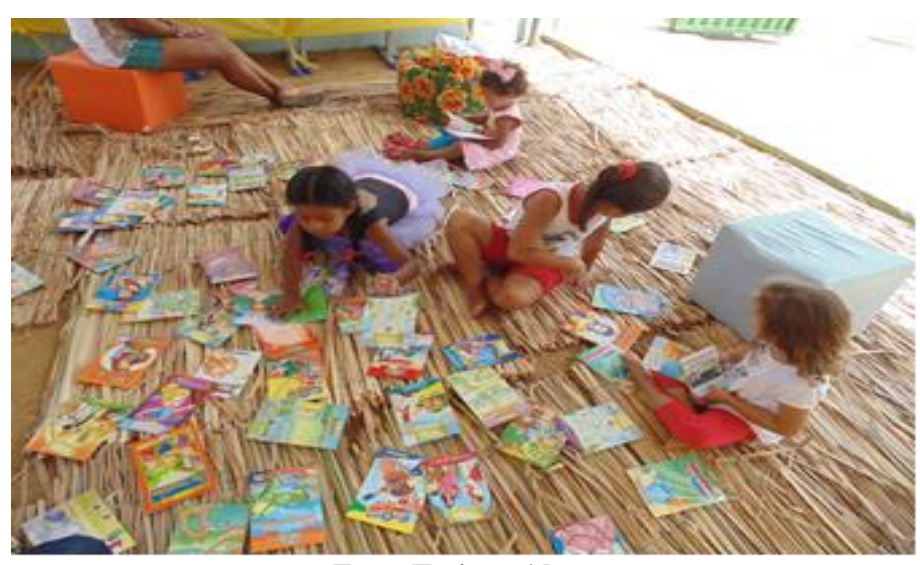

Foto: Tatiane Alves

As práticas de leitura na sala de aula e na biblioteca foram alvo da pesquisa bibliográfica para fins de comparação com as atividades desenvolvidas no universo pesquisado.

\footnotetext{
${ }^{3}$ Vale ressaltar que o projeto obedeceu a todos os procedimentos éticos relacionados à pesquisa com seres humanos, sendo submetido e aprovado pelo Comitê de Ética da Pesquisa (CEP) - UNEB. Ademais, foram utilizados, durante as entrevistas, os Termos de Consentimento Livre e Esclarecido (TLCE).
} 


\section{Análise documental}

Além das técnicas de coleta de dados descritas anteriormente, que envolvem diretamente as pessoas, a pesquisa fez uso de documentos, tais como registros que se referem aos dados de pessoas envolvidas com a biblioteca e ao fenômeno estudado. Esse instrumento foi utilizado para compreender melhor o funcionamento das bibliotecas, traçar o perfil dos usuários e entender as políticas públicas a que as unidades tinham acesso.

Para fins desta pesquisa, foram considerados como documentos, não apenas os escritos utilizados para esclarecer determinado evento, mas qualquer objeto que pudesse contribuir para a investigação de determinado fato ou fenômeno. (GIL, 2008). De acordo com Ludke e André (1986, p.38), a análise documental se constitui em "uma técnica valiosa de abordagem de dados qualitativos, seja complementando informações obtidas por outras técnicas, seja desvelando aspectos novos de um tema ou problema". Os documentos são usados no sentido de contextualizar o fenômeno, explicitar suas vinculações mais profundas e completar as informações coletadas através de outras fontes. (ANDRÉ, 2010).

Foi utilizada, na análise documental dessa investigação, uma combinação de documentos, o que permitiu recuperar as atividades desenvolvidas na biblioteca além de documentos oficiais, tais como: Portarias e Resoluções do PNBE, Lei 12.244/2010, Resolução n.7 de 2015 da CEE-BA, Livro de tombo, fichas de usuários, formulários da biblioteca, regulamentos, manuais, plano de ação da biblioteca, controle de acessos, ficha de empréstimo, cartazes, folders, projetos de leitura.

Além dos instrumentos expostos anteriormente, houve a necessidade de traçar o estado da arte sobre biblioteca escolar, no sentido de conhecer as tendências e lacunas das pesquisas já efetuadas para melhor alicerçar este estudo.

\section{Estado da arte}

O estado da arte foi utilizado por entender que ele possibilita a efetivação do balanço da pesquisa de uma determinada área. Romanowski e Ens (2006, 39 p.) esclarecem sobre sua contribuição.

Os estados da arte podem significar uma contribuição importante na constituição do campo teórico de uma área de conhecimento, pois procuram identificar os aportes significativos da construção da teoria e 
prática pedagógica, apontar as restrições sobre o campo em que se move a pesquisa, as suas lacunas de disseminação, identificar experiências inovadoras investigadas que apontem alternativas de solução para os problemas da prática e reconhecer as contribuições da pesquisa na constituição de propostas na área focalizada.

Segundo Chartier (2001 apud Aliaga e Silva 2012, p.60), a produção do estado da arte em uma pesquisa envolve,

de um lado, o "temor à perda", o desejo de conservar, proteger, guardar, de forma que os escritos encontrem-se organizados e disponíveis para aqueles que deles necessitem como também remete ao "temor do excesso", ao esforço de escolher e selecionar aquilo que é mais relevante diante da impossibilidade, dentro da cultura escrita, de cada indivíduo apropriar-se de tudo que é produzido.

Segundo Ferreira (2002), as pesquisas de "estado da arte" ou "estado do conhecimento", nos últimos 15 anos no Brasil, parecem trazer em comum o desafio de mapear e de discutir a produção acadêmica em diferentes campos do conhecimento, tentando responder que aspectos e dimensões vêm sendo destacados e privilegiados em diferentes épocas e lugares, de que formas e em que condições têm sido produzidas certas dissertações de mestrado e teses de doutorado, publicações em periódicos e comunicações em anais de congresso e seminários.

Vale ressaltar que existe uma clara diferença entre pesquisa bibliográfica e estado da arte. Corroborando com esse pensamento Aliaga (2013, p.61) esclarece, nas pesquisas bibliográficas, que "o pesquisador recorre aos bancos de teses e dissertações ou às revistas científicas com o intuito de buscar trabalhos já realizados que se aproximem e dialoguem com o seu objeto de pesquisa”. Já no caso da pesquisa estado da arte, ela "busca configurar a própria construção do corpus, uma vez que o conjunto de trabalhos encontrados dentro das delimitações impostas pelo pesquisador torna-se seu próprio objeto de pesquisa”. Assim, no estado da arte é analisado todo material produzido em determinada área, evidenciando novas ideias, tópicos específicos, enfoques escolhidos e métodos utilizados e tendências. Raras vezes um pesquisador iniciante consegue realizar um estado da arte, pois requer muita experiência no campo.

Dessa forma, para construir uma história da arte sobre biblioteca escolar, definiram-se como corpus da pesquisa os artigos de periódicos científicos, dissertações e teses. Foram analisados artigos de periódicos científicos por entender que essas publicações "constituem um dos mais eficientes meios de registro e divulgação de pesquisas, estudos originais e outros tipos de trabalho intelectual" (CUNHA, 2001, 
p.16). Já as dissertações e teses foram escolhidas como fonte de informação devido a sua importância no meio acadêmico por apresentarem uma pesquisa original sobre determinado tema.

Optou-se por analisar a história da arte tanto quantitativa quanto qualitativamente, ou seja, na primeira perspectiva, indicando quantos trabalhos foram produzidos, quais as regiões com maior incidência dentre outros aspectos e na segunda, tratando sobre as facetas da biblioteca escolar estudada nas pesquisas.

Assim foram analisados artigos científicos (das áreas de Educação e Ciência da Informação/Biblioteconomia), dissertações e teses nas bases: Literatura Brasileira em Biblioteca Escolar (LIBES), mantida pelo Grupo de Estudos em Biblioteca Escolar (ECI) da Universidade Federal de Minas Gerais; Banco de Teses da Coordenação de Aperfeiçoamento de Pessoal de Nível Superior (CAPES); Biblioteca Digital Brasileira de Teses e Dissertações do Instituto Brasileiro de Informação em Ciência e Tecnologia (IBICT).

A produção científica sobre biblioteca escolar foi analisada utilizando categorias como periódicos com maior produção, evolução temporal e temáticas mais abordadas. No que tange aos periódicos nacionais da área de Ciência da Informação/ Biblioteconomia sobre biblioteca escolar, foi possível identificar quais os assuntos privilegiados nas produções, bem como analisar os aspectos e as dimensões que vêm sendo destacados nas discussões acerca do tema. Para tanto, foi utilizado um recorte temporal, buscando as publicações mais recentes do período de 2010 a 2015, publicada em dez periódicos que compuseram o corpus do Estado da arte.

Como estratégia de busca, foi utilizado o termo biblioteca escolar sem discriminar o campo de busca. Dessa forma, o termo poderia aparecer tanto no título quanto nos assuntos ou nas palavras-chave. Foram aplicados alguns filtros na busca, tais como: a área do conhecimento, o espaço temporal e o idioma (português).

Para o levantamento da produção de dissertações e teses, após vasta pesquisa em diversas bases de universidades, optou-se por considerar o resultado de três fontes de informação que concentram, no seu acervo, as produções acadêmicas de diversas instituições, o que atenderia de forma eficaz à busca para compor este estado da arte. Sendo assim, elegeram-se três bases: Literatura Brasileira em Biblioteca Escolar (LIBES), mantida pelo Grupo de Estudos em Biblioteca Escolar - ECI - UFMG; Banco de Teses da Coordenação de Aperfeiçoamento de Pessoal de Nível Superior (CAPES); 
Biblioteca Digital Brasileira de Teses e Dissertações do Instituto Brasileiro de Informação em Ciência e Tecnologia (IBICT). Foram feitos recortes como estratégia de busca, destacaram-se alguns parâmetros, a saber: i) foram determinadas as seguintes palavras-chave: biblioteca escolar, bibliotecas escolares, biblioteca da escola, biblioteca na escola; ii) a pesquisa foi realizada dentro do espaço temporal de 2010 a 2015 buscando identificar as tendências das pesquisas desenvolvidas no Brasil neste período.

A primeira estratégia para a construção dos dados desta pesquisa foi partir do Banco de Teses da Coordenação de Aperfeiçoamento de Pessoal de Nível Superior $(\mathrm{CAPES})^{4}$, que permite consulta por meio dos campos de autoria, assunto, instituição, área de conhecimento, programa de pós-graduação, nível do curso e ano de produção. Como o termo "biblioteca" é muito genérico, optou-se por fazer a busca avançada, concentrando-se no campo do título. O segundo momento da pesquisa se concentrou na Biblioteca Digital Brasileira de Teses e Dissertações do Instituto Brasileiro de Informação em Ciência e Tecnologia $\left(\right.$ IBICT) ${ }^{5}$. Existe a possibilidade, nessa biblioteca digital, de filtrar a busca por instituição, nível do curso, assunto, autor, idioma e a no de publicação. E, por fim, no terceiro momento, foi realizada a busca na Literatura Brasileira em Biblioteca Escolar (LIBES) ${ }^{6}$. Como essa base é específica de biblioteca escolar foram utilizados como critérios de busca, o nível do curso e espaço temporal já definido anteriormente.

\section{Considerações finais}

O proceder metodológico representa a escolha do caminho a ser trilhado combinando métodos, abordagens e tipologias de pesquisa. $\mathrm{Na}$ produção do conhecimento compreender e interpretar fenômenos, a partir de seu contexto é tarefa importante para que o pesquisador se aproxime da realidade estudada. Para Silva (2005), "a metodologia tem como função mostrar a você como andar no "caminho das pedras' da pesquisa, ajudá-lo a refletir e instigar um novo olhar sobre o mundo: um olhar curioso, indagador e criativo".

Desse modo, o caminho trilhado nos levou a combinação das abordagens quantitativas e qualitativas que possibilitou congregar controle dos vieses com

\footnotetext{
${ }^{4}$ Banco da Capes - Disponível em: http://bancodeteses.capes.gov.br/

${ }^{5}$ Biblioteca Digital do Ibict - Disponível em: http://bdtd.ibict.br/vufind/

${ }^{6}$ Literatura Brasileira em Biblioteca Escolar - LIBES - Disponível em: libes.eci.ufmg.br
} 
compreensão das perspectivas dos agentes envolvidos no fenômeno, ou seja, apresentou a visão de alunos, professores e demais funcionários sobre as práticas de leitura desenvolvidas naquele ambiente e a relação dessas com as políticas públicas.

A escolha pelo estudo de caso permitiu a análise profunda das unidades educacionais pesquisadas, possibilitando o exame detalhado do ambiente, dos sujeitos e em especial das práticas letradas aí desenvolvidas.

Por fim, percebemos por meio dos instrumentos de construção de dados utilizados que somente a criação de políticas públicas não é suficiente para a democratização da leitura. Assim entendemos que boas práticas já se iniciaram no ambiente dessas bibliotecas com apoio das políticas públicas, para que as bibliotecas se tornem um espaço de aprendizagem contínuo e dinâmico. No entanto vale ressaltar que, além de uma capacitação e avaliação periódica sobre essas políticas, é necessário maior entrosamento dos atores envolvidos. A união entre professor e responsáveis pela biblioteca pode maximizar o uso das obras tanto para efetivar as práticas pedagógicas, quanto para possibilitar a democratização da leitura.

\section{Referências bibliográficas}

ALIAGA, Renata. A biblioteca escolar na produção acadêmica sobre leitura: movimentos, diálogos, aproximações. Dissertação (Mestrado), Campinas, SP, 2013.

ALIAGA, Renata; SILVA, Lilian Lopes Martin da. Pesquisas sobre leitura e biblioteca: um percurso de investigação e seus primeiros achados. In: CONGRESSO INTERNACIONAL DE LEITURA E LITERATURA, 3, 2012, Porto Alegre. Anais eletrônicos...Porto Alegre: Edipucrs, 2012. Disponível em: ebooks.pucrs.br/edipucrs/anais/IIICILLIJ/Trabalhos.

ANDRÉ, Marli. Etnografia da prática escolar.17. ed. Campinas: Papirus, 2010. 128 p. (Prática pedagógica)

CHARTIER, Roger (2001). Cultura escrita, literatura e história: conversas de Roger Chartier com Carlos Aguirre Anaya, Jesús Anaya Rosique, Daniel Goldin e Antônio Saborit. Porto Alegre: Artmed.

CUNHA, Murilo Bastos da. Para saber mais: fontes de informação em ciência e tecnologia. Brasilia: Briquet de Lemos, 2001. 168p.

GIL, Antonio Carlos. Métodos e técnicas de pesquisa social. 6 ed. São Paulo: Atlas, 2008. 
GOLDENBERG, Mírian. A Arte de Pesquisar: como fazer pesquisa qualitativa. Rio de Janeiro: Record, 2004.

LUDKE, Menga; ANDRÉ, Marli E. D. A. Pesquisa em educação: abordagens qualitativas. São Paulo: EPU, 1986.

MINAYO, Maria Cecília de Souza; SANCHES, Odécio. Quantitativo-qualitativo: oposição ou complementaridade? Cad. Saúde Públ., Rio de Janeiro, 9 (3): 239-262, jul/set, 1993.

OLIVEIRA, Maria Marly de. Como fazer pesquisa qualitativa. 5 ed. Petropolis: Vozes, 2013.

ROMANOWSKI, Joana Paulin. ENS, Romilda Teodora. As pesquisas denominadas do tipo "estado da arte" em educação. Dialogo Educação, Curitiba, v.6, n.19, set/dez. 2006.

SILVA, Edna Lúcia da. Metodologia da pesquisa e elaboração de dissertação. 4 ed. rev. atual. Florianópolis:UFSC, 2005. 138p.

YIN. Robert K. Estudo de caso: planejamento e métodos. 4 ed. Porto Alegre: Bookman, 2010. 